\title{
3 Von der digitalen Apathie zur digitalen Empathie - Wege in eine patientenzentrierte Informationskultur
}

\author{
Joss Hertle
}

\section{Der Patient von früher - Eine aussterbende Art}

Er ging bei Beschwerden zum Arzt, vertraute auf Diagnose und empfohlene Behandlung und nahm die verordneten Medikamente ein. Wenn er seine Gesundheit vorbeugend unterstützen wollte, suchte er dafür in der Regel den Apotheker seines Vertrauens auf und ließ sich dort beraten - der Patient von früher war leicht zu durchschauen und hat das Leben der Akteure in der Healthcare-Branche dadurch einfach gemacht. Aber er gehört einer aussterbenden Art an. Seinen Platz nimmt zunehmend eine neue Spezies von Patienten ein.

Der Patient von heute ist mit dem von früher nicht mehr zu vergleichen: Die bisherige Arzthörigkeit wurde von einem eigenständigen, souveränen Verhalten abgelöst. Der mündige Patient, so ließe sich die neue „Spezies“ beschreiben, sammelt selbst Informationen, geht mit seiner Gesundheit eigenverantwortlich um und tritt dem Arzt als gleichgestellter Partner gegenüber. Dementsprechend hat sich auch seine patient journey, die Wissensreise des Patienten durch das Web, grundlegend geändert. Erster Ansprechpartner in Gesundheitsfragen ist nun in der Regel Dr. Google, nicht mehr der Arzt oder Apotheker. Beide Gatekeeper der Gesundheitswelt von gestern haben an Macht eingebüßt und auch an Vertrauen verloren. Der freie Zugang zu relevanten Gesundheitsinformationen verändert die Selbstwahrnehmung des Patienten vom hörigen Konsumenten zum Dialogpartner auf Augenhöhe mit Arzt und Apotheker. Der Patient hat die Macht. Es überrascht dabei kaum, dass man bei der Netzrecherche auf einigen Gesundheitsseiten den Tipp findet, bei einer ernsthaften Diagnose mehrere Ärzte zu konsultieren und nicht bei der Stichprobe $\mathrm{n}=1$ zu verharren. 
Heute wartet im Web - im Idealfall - entscheidungsrelevanter, hochwertiger Content darauf, den Suchenden auf seiner Reise weiterzuführen und wichtige Informationen zu vermitteln. Zahlreiche Apps unterstützen den Patienten zusätzlich - sowohl begleitend bei einer Erkrankung als auch als eigenständiges Instrument, um zu mehr Gesundheit zu finden. Mit den gesammelten Informationen setzt sich der mündige Patient kritisch auseinander, bildet sich eine Meinung. Anschließend geht er zum Arzt oder sucht eine Apotheke auf - im Internet oder auch stationär - um dort, in Zusammenarbeit mit den Behandlern, die optimale Lösung für seine Gesundheit zu finden. Die Gesundheitssuche ist dabei nicht linear, der Patient springt in seinen Entscheidungsphasen in puncto Bedürfnisanalyse, Evaluation bzw. Transaktion und Post-Transaktion hin und her. Das macht die Patient Journey für alle Wertschöpfungs-Beteiligten in der Gesundheitsindustrie umso komplexer.

\section{Was bedeutet dieser Ist-Zustand für das Digitalverständnis von Ärzten, Apothekern oder der Pharmaindustrie?}

\section{Digitale Transformation - Digitale Empathie}

Im Mittelpunkt der Digitalisierungsprozesse kann nur der Patient stehen. Er ist der Dreh- und Angelpunkt und hat das Sagen, was seine Gesundheit, sprich die Behandlung und Vorsorge angeht. Dieses eigenverantwortliche Handeln und Mitdenken des Patienten ist wünschenswert und auch gut so, denn viel zu oft liegt der Arzt mit seiner ersten Diagnose nicht richtig. Informationen, die dem Patienten im Zuge der Digitalisierung zugänglich werden, können ihn für seine Gesundheit und mögliche Symptome sensibilisieren - und so zu einer schnelleren und genaueren Diagnose beitragen. Ärzte stehen heute Patienten gegenüber, die sich bereits eine Meinung gebildet haben. In Anbetracht dieses Faktums in nostalgische Schwermut zu verfallen und diesen ,neunmalklugen, neumodernen Patienten' zu verteufeln, ist ein durchaus verständliches, aber nicht zielführendes Ressentiment. Abwehr- und Verweigerungshaltungen aufseiten der Gesundheitsbranche sind mitunter nicht unbegründet und durchaus nachvollziehbar, bergen jedoch zugleich die Gefahr, die Bewegung von produktzentrierter zu patientenzentrierter Gesundheitskultur zu unterschätzen. Gesundheits-Gatekeeper brauchen für die digitale Orientierung eine andere Einstellung, eine Art Digitale Empathie, um Ansichten und angelerntes Wissen ihrer Patienten zu verstehen und sie ernst zu nehmen, zum anderen aber auch, um selbst verständlich erklären zu können, warum sie im Einzelfall anderer Meinung sind.

Grundsätzlich ist es sinnvoll, das eigene private Digitalverhalten in die tägliche Praxis (im doppelten Wortsinn) zu übertragen. Um eine Verhaltensänderung herbeizuführen, sollten sich Ärzte, Apotheker und die Pharmaindustrie bewusst werden, dass wir uns im Beruflichen zu oft von der vorgezeichneten, bequemen Macht des Inkrementellen erfassen lassen - wir entwickeln Standpunkte, Erfahrungen und Glaubenssätze. Es werden Erfolge verbucht und diese erfolgreichen Verhaltensweisen werden abgespeichert. Für das Verständnis der Möglichkeiten und Chancen der Digitalisierung, durch die der Patient die Gesundheitsparteien vor sich her treibt, braucht es aber eine Vorstellung von den Möglichkeiten, die die Digitalisierung eröffnet, z.B. 
in der Kommunikation. Dafür muss die rote Linie der Glaubenssätze überschritten werden. Für das Verständnis der Digitalen Möglichkeiten ist das „Neu-Gierig-Sein“ (Sprenger 2015) eine unerlässliche Kerntugend. Es gilt, die gegebenen Tools, Geräte und Dialogmöglichkeiten zu nutzen, um sich ins Bewusstsein des Patienten zu bringen. Dafür müssen sich die Gesundheitsakteure von der Macht des Objektiven lösen und die Sicherheit des Analogen aufgeben.

\section{Erfolgreiche digitale Transformation erfordert das Loslassen von be- währten analogen Glaubenssätzen. Es erfordert eine neue Geisteshal- tung, die Digitale Empathie.}

Die Zukunft gehört der Kommunikation zwischen den Gatekeepern und dem Patienten, gehört den neuen Dialogformen, z.B. dem relevanten Beratungs-Content, ob via Internet oder Apotheker und Ärzte. Informationen auf Webseiten sollen den User abholen und ihm bei der Entscheidungsfindung helfen. Für die Verantwortlichen bedeutet das, dass sie sich in den Nutzer hineinversetzen müssen. Was für Bedürfnisse hat er? Was für eine Werteorientierung besitzt er? Welcher Content bietet ihm eine wirkliche Hilfe? Und welche Motivation treibt ihn an? Dies alles gilt es, in der modernen Zeit besser zu verstehen. Denn: Dem Patienten allein gehört seine Gesundheit. Er will verstanden, aber auch beraten werden. Wer das einsieht, dem bietet die Digitalisierung eine riesige Chance.

\section{Potenziale der Patientenzentrierung}

Der digitale Wandel bietet den Akteuren die Chance, Teil der digitalen Wertschöpfung des Patienten zu werden, sich am Markt neu und besser zu positionieren und gleich zu Beginn der patient journey in das direkte Blickfeld des Patienten zu gelangen. Dafür ist jedoch ein wichtiger Schritt notwendig: Alle Gesundheitsakteure - Ärzte, Apotheker und vor allem Pharmafirmen - müssen eine neue Denkweise erlernen. Sie müssen immer wieder neu zupacken, schnell lernen und stets optimieren. Das „Fertige“, das einmal entworfene und dann gültige, gibt es nicht mehr. Und auch die klassischen Zielgruppen gibt es nicht mehr - sie können zusammen mit überholten Dialogformen und dem Kampagnen-Denken getrost in die Schublade gelegt werden. Das Alter als ehemals klassisches Kriterium zur Zielgruppendefinition im Pharma-Marketing z.B. verliert zunehmend an Bedeutung - vor allem was die Wahl der Werbemittel und Kanäle betrifft. Marketeers, die einen demografischen Trennstrich zwischen Print-bzw. TV- und Web-Werbung ziehen, verkennen die Tatsachen: Menschen jeden Alters nutzen das Internet für Ihre Bedürfnisevaluation und können dort gezielt „abgeholt“ werden. Gerade im Personenkreis der über 6o-Jährigen sind bereits circa 50\% aktiv mit Smartphone und Co. im Internet unterwegs - Tendenz steigend.

Analysen von Google-Suchanfragen zeigen hierbei, wie stark das Bedürfnis der Patienten ist, sich im Internet über Gesundheitsthemen zu informieren. Pro Tag liegt die Zahl der Anfragen zu Gesundheitsthemen bei ca. 20 Millionen. Digitale Medien bieten dabei sowohl Nutzern als auch Anbietern den bedeutenden Vorteil, dass sie 
3 Von der digitalen Apathie zur digitalen Empathie -

Wege in eine patientenzentrierte Informationskultur

jederzeit abrufbar und - vor allem im Vergleich zu Print-Medien - leicht auf aktuellem Stand zu halten sind.

Schon zu Beginn der patient journey können Firmen dafür sorgen, durch professionelle SEA- und SEO-Maßnahmen auf Seite eins der Suchergebnisse zu landen und den Patienten auf ihre Webseite zu lotsen. Nun muss der Content folgen, mit hohem Informationsgehalt, professionell erstellt, gleichzeitig verständlich für den Patienten und mit erforderlicher Informationstiefe. Unternehmen können hierbei verschiedene Tools nutzen, um den Patienten besser zu verstehen, seine Aktionen online nachzuverfolgen und so noch zielgerichteter auf ihn eingehen zu können.

Um den Wandel erfolgreich mit zu vollziehen, sollten die Akteure im HealthcareSektor damit anfangen, Digitale Empathie zu entwickeln. Nur durch Spezialisierung, Differenzierung und den Ausbau ihrer digitalen Kommunikationskanäle werden Apotheker, Ärzte und Pharmaunternehmen langfristig einen entscheidenden Sichtbarkeitsvorteil gegenüber der Konkurrenz haben.

Darauf kommt es an: Kompetenzen vereinen, flexibel reagieren und Fehler nicht krampfhaft vermeiden, sondern zur Weiterentwicklung nutzen.

\section{Die Zukunftsmusik in der Healthcare-Branche}

Schon heute bereichern zahlreiche Apps, Fitness-Tracker und andere Anwendungen das Angebot für die Patienten, ihre Gesundheit im Auge zu behalten und zu optimieren. Sie ermöglichen es, bei chronischen Erkrankungen den Verlauf unkompliziert und ohne großen Aufwand zu überwachen und so eine Veränderung bereits festzustellen, bevor sie sich in Symptomen bemerkbar macht. Parallel dazu helfen entsprechende technische Anwendungen dabei, viele Faktoren gleichzeitig im Blick zu behalten, die für die Erhaltung der Gesundheit und Fitness wesentlich sind: Schlafrhythmus, Bewegung und Ernährung sind nur eine Auswahl Gesundheitsdaten, die durch solche Anwendungen gesammelt und auch analysiert werden können.

Derzeit laufen bereits einige aussichtsreiche Testphasen von closed-loop-Systemen, die es Patienten ermöglichen, per Smartphone Medikamente zu dosieren und automatisch nachzubestellen. Hier entfernt sich die zentrale Weiche der Wertschöpfungskette von der Frage Wer verkauft die Medikamente? hinzu Wer entwickelt die Systeme? und vor allem Wer beliefert den Patienten? Dabei ist es gleichgültig, ob derjenige global player, der sich dieser lukrativen Aufgabe annimmt, ein antikes Geschlecht von Kriegerinnen im Namen trägt - der Markt wird sich bewegen und aus Perspektive der Aktanten im Gesundheitswesen wäre es fatal, sich auf bestehende rechtliche Regelungen oder den Gesetzgeber zu verlassen.

Insgesamt wird die Entwicklung im Healthcare-Bereich darauf zusteuern, dass der Patient immer mehr Einsicht in und auch Einfluss auf Vorsorge und Behandlung erlangt. Er gewinnt dadurch zunehmend an Wichtigkeit und behauptet - zu Recht seinen Platz im Fokus des Pharma- und Healthcare-Marketings. 


\section{Einführung}

\section{Literatur}

Heckmann D (2015) Digitale Empathie“ - wie kommt mehr Gerechtigkeit ins Internet, br-alpha Keese C (2016) Silicon Germany - wie wir die digitale Transformation schaffen, Knaus Verlag, München Schäffler B (2016) Smart Healthcare. Die Zukunft beginnt heute. URL: http://www.trendone.com/fileadmin/ Redaktion/PDF/HCM_9_2016.pdf (zugegriffen am 25. April 2017)

Sprenger RK (2015) Das Prinzip Selbstverantwortung, Campus Verlag, Frankfurt a.M. 